\title{
Analyse statistique et probabiliste de la corrosion de quais sur pieux de l'estuaire de la Loire
}

\author{
Alexandre Clément* ${ }^{* \mathrm{a}}$, Franck Schoefs ${ }^{* \mathrm{~b}}{ }^{\mathrm{b}}$ Jean-Bernard Memet ${ }^{*{ }^{*}}$, \\ Anthony Nouy \\ * Université de Nantes, CNRS, Institut de Recherche en Génie Civil et \\ Mécanique (GeM), UMR 6183, Faculté des Sciences et des \\ Techniques, 2 rue de la Houssinière, Nantes, F-44000 France. \\ ** Arc'antique, Rue de la Haute Forêt, 44000 Nantes \\ a) Doctorant; alexandre.clement@univ-nantes.fr \\ b) Maître de conférences; franck.schoefs@univ-nantes.fr \\ c) Ingénieur de recherche; arcantique.recherche@wanadoo.fr \\ e) Maître de conférence ; anthony.nouy@univ-nantes.fr
}

\section{Résumé}

La corrosion d'ouvrages métalliques en mer ou estuaire constitue une source de désordres majeurs dans leur comportement en service et nécessite d'être caractérisée et prise en compte lors de calculs mécaniques ayant comme paramètre l'épaisseur de matière. Les mesures d'épaisseurs résiduelles effectuées par ultra-son permettent d'établir des profils de perte de matière et d'identifier les zones les plus sollicitées par la corrosion. La caractérisation de l'erreur de mesure permettra, elle, de réaliser, dans le futur, une étude mécano-probabiliste des quais inspectés et d'optimiser encore plus leur inspection.

\begin{abstract}
$\underline{\text { Abstract }}$
Corrosion of metallic structures in sea or estuary area represents a great source of problems in their behaviour during their service life and needs to be characterised for mechanical calculations which take into account the loss of thickness. Ultrasonic residual measurements allow us to determine profiles of loss of thickness and to identify the areas which are the most affected by corrosion. The characterization of the error of measurement will also help us to make, in the future, a mechanical and probabilistic study of the wharves to optimize their inspections.
\end{abstract}

\section{Mots clés}

corrosion, quais sur pieux, mesures ultrasons, perte de matière, erreur de mesure 


\section{Introduction}

Des campagnes d'inspections CND (Contrôle Non Destructif) sont usuellement réalisées afin de déterminer les pertes d'épaisseur des pieux à différentes cotes et pour différentes expositions aux efforts hydrodynamiques. Les protocoles mis en place par le CETMEF (Centre d'Etudes Maritimes et Fluviales) permettent alors d'avoir une représentation spatiale de la perte de matière sur les pieux des quais inspectés. A partir des différentes campagnes d'inspections, menées dans l'estuaire de la Loire entre Nantes et Montoir, on constate que les sites les plus affectés par les phénomènes de corrosion se trouvent à l'embouchure de la Loire (site de Montoir). C'est pourquoi nous étudions ici les données d'inspections issues des postes 2 et 3 du TMDC (Terminal à Marchandises Diverses et Conteneurs) du site de Montoir commandées par le Port Autonome de Nantes Saint-Nazaire.

Cet article, s'inscrivant dans le cadre du projet européen MEDACHS (Marine Environment Damage to Atlantic Coast Historical and transport works or Structures), présente une analyse statistique de la répartition des pertes d'épaisseur sur les pieux et les résultats d'une étude sur les distributions de l'erreur de mesure. Une comparaison sera faite entre les deux campagnes de mesures sur les deux postes et nous montrerons par quelle loi nous avons ajusté cette distribution (la loi normale ne s'avérant pas satisfaisante).

\section{$\underline{\text { 2. Description des quais sur pieux }}$}

Les deux quais étudiés lors de cette étude sont situés sur la commune de Montoir en Loire-atlantique, à deux kilomètres en aval du pont de Saint-Nazaire sur la rive Nord de l'embouchure et sont exploités par le port autonome de Nantes SaintNazaire. Il s'agit des postes 2 et 3 du TMDC. Ces deux postes ont été construit en 1983 (poste 2) et en 1984 (poste 3) et ont été inspectés par l'entreprise Tétis entre octobre 2004 et janvier 2005 selon un protocole de mesure par ultra-son préconisé par le CETMEF.

\subsection{Présentation des postes 2 et 3 du TMDC}

Le poste 2 présente une longueur de 203,2 $\mathrm{m}$ et une largeur de $40,9 \mathrm{~m}$, il est composé de 32 pieux-tubes en acier (épaisseur nominale $(10 / 14 \mathrm{~mm})$ ) remplis de béton et la peinture anticorrosion est une peinture bitumeuse. On notera que le poste 2 comprend quatre files principales (A, C, E et F) qui comptent sept pieux et deux files secondaires (B et $\mathrm{D})$ ne comprenant que deux pieux chacune. La file $\mathrm{A}$ est la file la plus proche de l'estuaire et la file $\mathrm{F}$ la plus proche de la berge.

Le poste 3 présente lui une longueur de $253,2 \mathrm{~m}$ et une largeur de $40,9 \mathrm{~m}$, il est composé de 30 pieux-tubes en acier (épaisseur nominale $(10 / 12 \mathrm{~mm})$ ) remplis de béton et la peinture anticorrosion utilisée lors de l'installation des pieux est une peinture bitumeuse. 
Les deux postes sont situés l'un à côté de l'autre et le poste 2 se situe plus en amont de l'estuaire. Leurs dates de construction proches et leurs fortes similitudes, du point de vue géométrique et sollicitations environnementales, permettent de comparer les différents résultats obtenus pour cette étude.

\subsection{Protocole de mesure}

Le protocole de mesure suivi par l'entreprise Tétis consiste à effectuer des mesures, d'épaisseurs résiduelles par sonde ultra-son à différentes cotes sur quatre zones (points cardinaux) d'une section du pieu. A partir de ces mesures d'épaisseur résiduelle et, sachant les épaisseurs nominales (aux tolérances de laminage près) des pieux lors de leur installation (données sur les plans de construction), il nous est possible de déterminer la perte de matière due principalement à la corrosion. Pour un pieu et à une cote donnée, 12 mesures sont effectuées réparties en 3 mesures « horaires » pour chacun des 4 zones cardinales de $40 \mathrm{~mm}$ de diamètre (cf figure 1). Il est important d'effectuer des mesures pour chacun de ces points cardinaux afin de ne pas s'affranchir de certains effets locaux influents sur le développement de la corrosion (courants, vents, etc.) et d'avoir une représentation "spatiale » sur la section d'un pieu. Cependant, il est évident que ces quatre seuls points ne suffisent pas à avoir une représentation de la perte de matière sur toute la section.

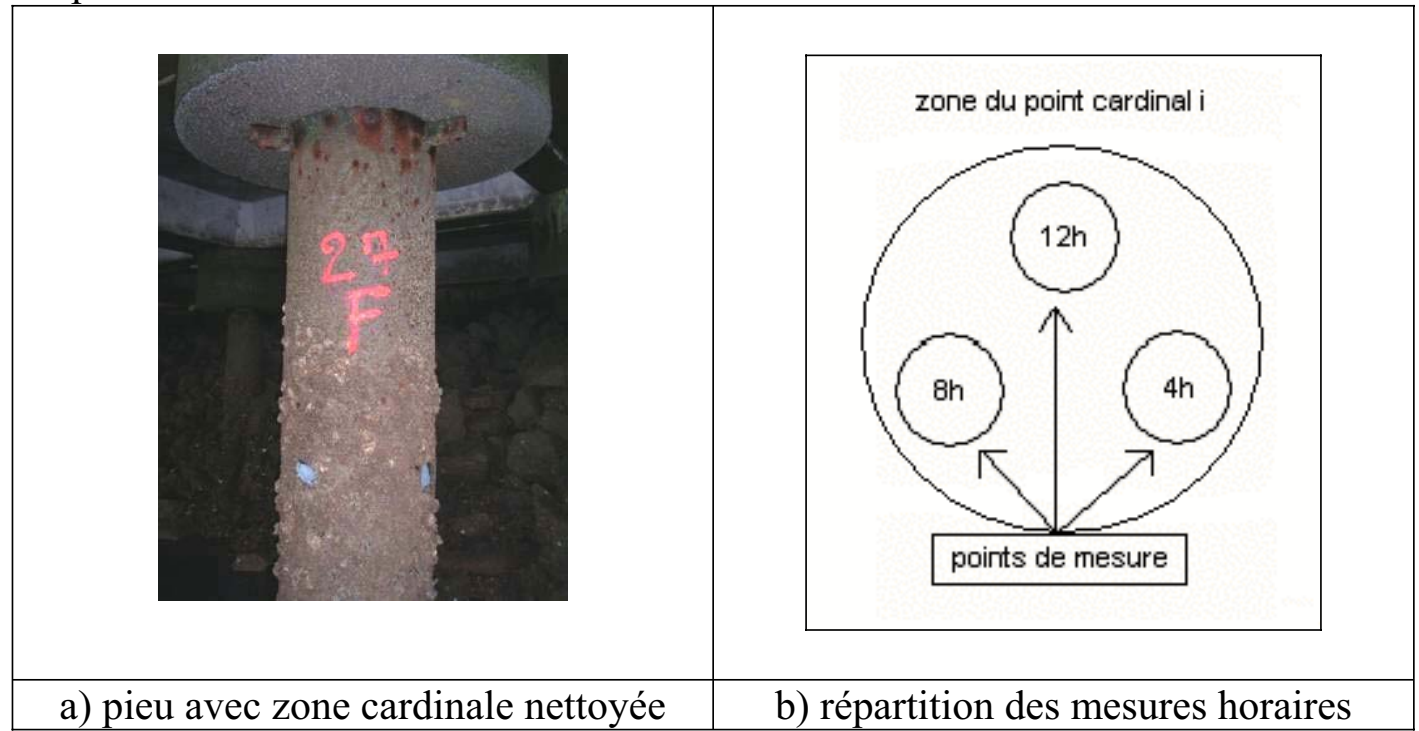

Figure 1 : exemple de pieu inspecté et répartition des zones de mesures horaires

\section{Analyse statistique des données de perte de matière}

Dans cette section nous allons regrouper de différentes manières les données collectées afin de dégager des tendances pour chaque file de pieux. On étudiera les données en présentant des profils de perte de matière en fonction de la profondeur : on regroupera, dans un premier temps, les données du poste 2 par point cardinal pour définir leur influence, puis, dans un second temps, par file, 
pour dégager des tendances sur les pertes de matière dues à la corrosion et comparer les données des deux postes.

\subsection{Analyse des différences entre points cardinaux}

Intéressons nous tout d'abord à l'influence des points cardinaux. Pour cela nous calculons les moyennes et écarts types des mesures pour chaque point cardinal d'une même file de pieux. Ainsi, nous regroupons les données horaires par point cardinal et, dans le même temps, ramenons les sept pieux d'une même file à un seul. Les moyennes et écarts types sont alors calculés à partir de 21 valeurs. On présente sur la figure 2 l'évolution de la moyenne par point cardinal en fonction de la profondeur pour la file A. La ligne bleue se situant à $+0.6 \mathrm{CM}$ représente l'interface entre la zone de marnage et la zone de basses eaux (ou zone d'immersion permanente). Cette limite est importante car elle constitue en général une zone de forte corrosion bien connue $\left(\mathrm{Memet}^{4}\right)$. Il est à noter que pour ces postes, il n'existe pas de mesure faite dans la zone d'embruns puisque lors d'une marée d'équinoxe de vives eaux les pieux sont tous totalement immergés. Cependant étant donné la rareté de ces marées extraordinaires la zone se situant à + 3.50 CM peut être assimilée à une zone d'éclaboussures.

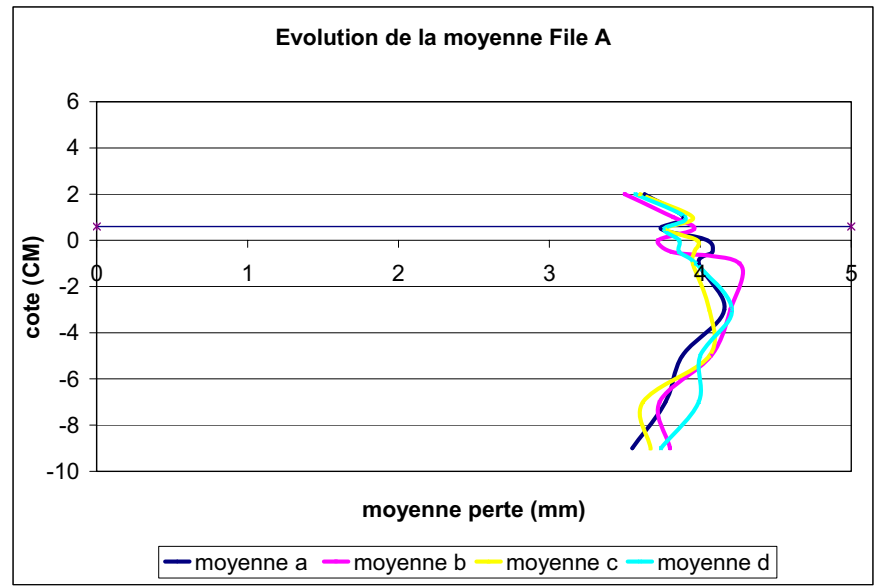

Figure 2 : évolution de la moyenne des pertes de matière relevées aux points cardinaux pour la file A (poste 2)

On constate sur cette figure que les profils sont semblables et que la perte de matière pour cette file se situe autour de $4 \mathrm{~mm}$, la valeur la plus élevée se situant à - $1 \mathrm{CM}$ pour le point cardinal $\mathrm{b}$ et à $-3 \mathrm{CM}$ pour les autres points cardinaux. Les autres files présentent des comportements très proches. Cependant, on a observé que les autres files du poste 2 sont nettement moins corrodées que la file A avec des pics de corrosion n'excédant pas les $2 \mathrm{~mm}$. Une étude sur les écarts types $\left(\right.$ Clément $\left.^{9}\right)$ conduit à des coefficients de variation assez faibles, compris entre 5 et $15 \%$, pour la file $\mathrm{A}$ et nettement plus importants pour les autres files (où la corrosion est bien moins importante) avec des valeurs comprises entre 30 et $40 \%$. En conclusion, les pertes de matière sont globalement les mêmes quels que soient les points cardinaux où les mesures ont été faites. Cependant il apparaît difficile de recommander de n'effectuer des mesures qu'à un seul d'entre eux tant les 
effets locaux influençant la corrosion sont nombreux et importants (effets visibles sur l'écart type).

\subsection{Comparaison entre les résultats des deux postes étudiés}

Dans le cas d'ouvrages maritimes de nature différente (comme un quai gabion et un quai sur pieux par exemple) et distants (conditions climatiques et hydrodynamiques très différentes), il est très difficile d'envisager une comparaison des effets de la corrosion. L'un des avantages de la campagne d'inspection réalisée pour le port autonome de Nantes Saint-Nazaire est le grand nombre de points communs entre les deux quais inspectés : leur géométrie, leur âge, leur fonctionnement (charges d'exploitations identiques), leur proximité (conditions climatiques et hydrodynamiques très proches). Ceci permet d'envisager une comparaison entre les résultats obtenus. Seul le paramètre important que constitue la nature de l'acier peut être différent. Nous présentons sur les figures 3 et 4 , les moyennes des pertes de matière obtenues pour les files $\mathrm{A}$ des postes 2 et 3 . On constate qu'il est possible de raisonner par file : c'est-à-dire que les pieux d'une même file présentent des tendances très similaires du point de vue de la corrosion et on peut ainsi regrouper les résultats.

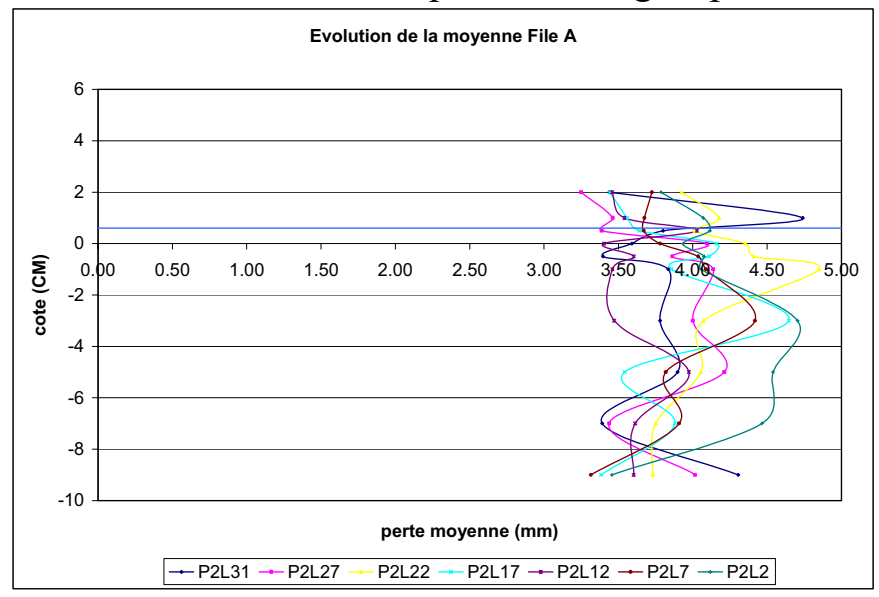

Figure 3 : moyennes des pertes de matière par pieu pour la file $\mathrm{A}$ du poste 2

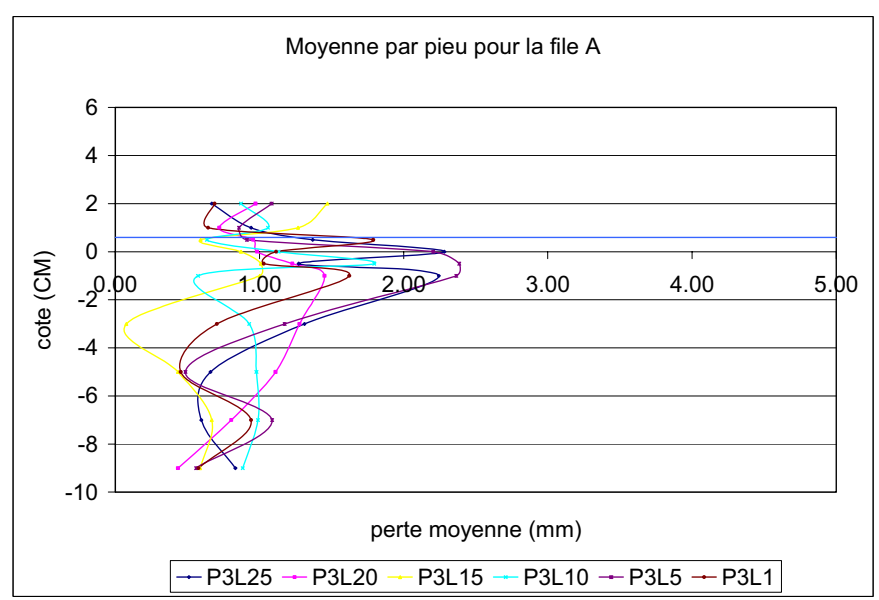


Figure 4 : Moyennes des pertes de matière par pieu pour la file A du poste 3

De plus on peut remarquer une grande différence de perte de matière entre les files A des deux postes : en effet la file $\mathrm{A}$ du poste 2 présente une perte moyenne de l'ordre de $4 \mathrm{~mm}$ alors qu'elle n'est que de $1.5 \mathrm{~mm}$ pour le poste 3 .

On présente maintenant sur les figures 5 et 6 les moyennes par files pour les deux postes : on notera que ces moyennes sont calculées pour 84 valeurs pour le poste 2 et 72 pour le poste 3 . On constate nettement un problème pour la file $\mathrm{A}$ du poste 2 : en effet celle-ci est nettement plus corrodée que les autres. Si ce phénomène pouvait paraître plausible en ne disposant que des résultats du poste 2 , il devient beaucoup discutable une fois les résultats du poste 3 obtenus (géométrie, fonction et conception similaires). D'ailleurs, les épaisseurs des pieux sont identiques à 1 $\mathrm{mm}$ près, entre deux mêmes files des deux postes (les files $\mathrm{C}$ par exemple). Or, d'après les plans fournis par le port de Nantes Saint-Nazaire, les pieux de la file A du poste 2 auraient une épaisseur de $14 \mathrm{~mm}$ alors que ceux de la file A du poste 3 auraient une épaisseur de $11 \mathrm{~mm}$ seulement. L'écart moyen de perte de matière entre les résultats des deux files A est d'environ $3 \mathrm{~mm}$.

Deux alternatives sont alors à envisager : soit les pieux de la file $\mathrm{A}$ du poste 2 ont une épaisseur de $11 \mathrm{~mm}$ soit ceux du poste 3 ont une épaisseur de $14 \mathrm{~mm}$. Le meilleur moyen de pouvoir savoir quelle hypothèse est juste, serait de trouver une zone saine sur l'un des pieux et de mesurer son épaisseur résiduelle, théoriquement proche de l'épaisseur initiale. Cette zone saine est délicate à localiser. Ceci souligne l'importance de la mesure initiale d'épaisseur des pieux. En ce qui concerne les autres files on constate que pour un même poste leurs comportements sont très proches (profils et valeurs), la file $\mathrm{C}$ (plus proche de l'estuaire) présentant, cependant, toujours une corrosion plus importante que la file $\mathrm{E}$. En revanche si on compare les résultats des files $\mathrm{C}$ et $\mathrm{E}$ entre les postes 2 et 3 , on peut observer une différence importante : pour le poste 2 , le pic de corrosion se situe en tête de pieu pour la cote $+5.0 \mathrm{CM}$ alors que pour le poste 3 le pic de corrosion se situe à la cote $+1.0 \mathrm{CM}$ (légèrement au dessus de l'interface) pour la file $\mathrm{C}$ et $+3.5 \mathrm{CM}$ pour la file $\mathrm{E}$. Ce phénomène de corrosion plus élevée en tête de pieu est étonnant, et si on en fait abstraction, on s'aperçoit que les pics de corrosion sont presque atteints aux mêmes cotes.

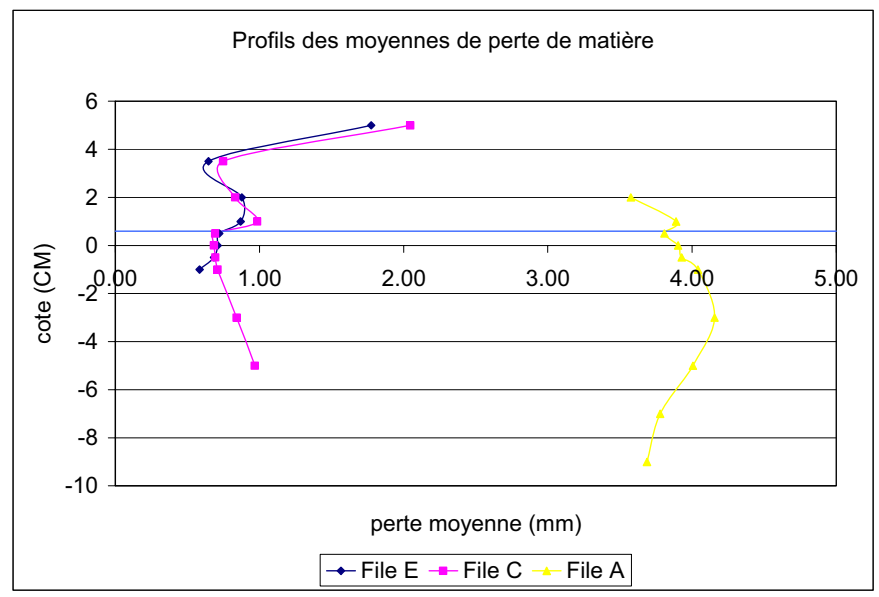

Figure 5 : moyennes des pertes de matière pour chaque file du poste 2 


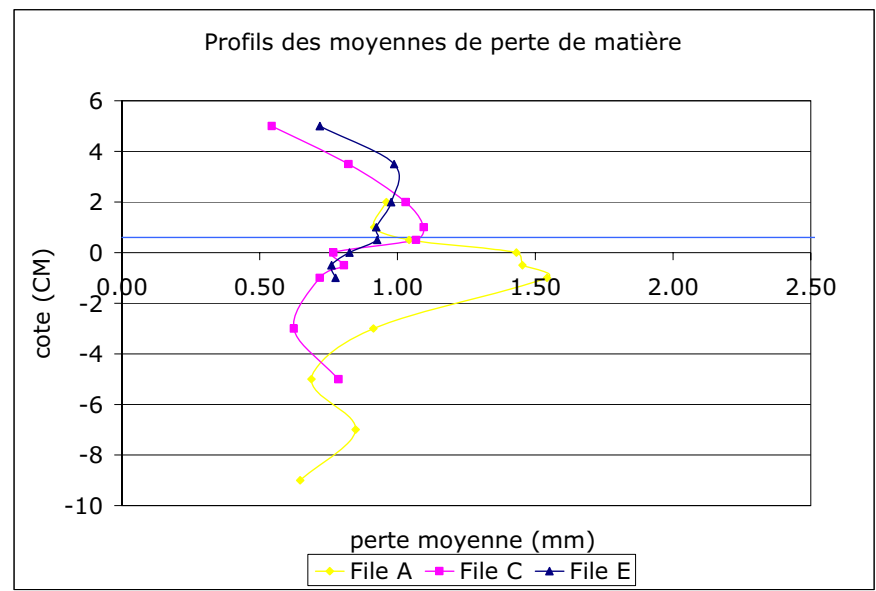

Figure 6 : moyennes des pertes de matière pour chaque file du poste 3

\section{Comparaison des erreurs de mesure des deux postes}

On s'intéresse dans cette section, aux erreurs de mesure obtenues pour les 2 postes et aux écarts constatés entre eux. L'erreur de mesure est une donnée importante qui sera prise en compte dans une modélisation mécano-probabiliste afin de prévoir le comportement de la structure soumise aux effets de la corrosion. Il est donc nécessaire de caractériser au mieux cette erreur et de pouvoir la représenter à l'aide d'une densité de probabilité soigneusement choisie.

L'erreur de mesure $\varepsilon$ est le « fil conducteur» de cette partie. Elle a été calculée comme suit :

$$
\varepsilon=\hat{S}-S
$$

où $\varepsilon$ est l'erreur de mesure,$\hat{s}$ est une mesure réalisée en un point cardinal et $s$ la taille réelle du défaut (la perte de matière réelle).

$\mathrm{Si} \hat{s}$ est donnée par les résultats de l'inspection, nous ne disposons pas, en revanche, de $s$. Pour l'obtenir, deux possibilités sont envisagées :

- $s$ peut être déterminée à l'aide d'une autre technique CND dont l'erreur de mesure serait plus faible qu'avec la technique ultra-son (inter calibration de techniques d'inspection);

- on fait l'hypothèse que $s$ est connue en supposant que certains paramètres ou propriétés (comme la moyenne) de la distribution de l'erreur sont connus.

A ce stade de l'étude, on considérera la seconde hypothèse en supposant que :

- l'erreur a une moyenne de 0 (pas de biais) ;

- la taille de défaut réelle est la moyenne obtenue sur les trois mesures horaires $(4 \mathrm{~h}, 8 \mathrm{~h}$ et $12 \mathrm{~h}) \ldots$;

- l'erreur de mesure ne dépend pas de la taille du défaut. 
On considère alors que chaque évènement ${ }_{\varepsilon_{i}}={ }_{s_{i}}^{\wedge} \bar{s}_{i}$, où $\bar{s}_{i}$ est la moyenne au point horaire $i$. Cette erreur, qui est en fait un estimateur de l'erreur, comprend à la fois l'incertitude sur l'appareil de mesure, l'influence du facteur humain et les difficultés liées aux conditions d'inspection. L'estimateur de l'erreur prend donc en compte la moyenne des trois mesures faites en un point cardinal i et les valeurs horaires mesurées en ce point cardinal.

\subsection{Etude de l'influence de la zone et de la file sur l'erreur de mesure}

On s'intéresse tout d'abord à l'influence de la zone de mesure sur cette incertitude. On distingue alors les mesures faites dans la zone de marnage et celles de la zone d'immersion permanente. Pour le poste 2 comme pour le poste 3 , on obtient des distributions très proches du type de celle présentée en figure 6 . Toutefois les statistiques sont légèrement différentes. Pour le poste 2, par exemple, on obtient dans la zone de marnage une moyenne de $0.0019 \mathrm{~mm}$ et un écart-type de $0.118 \mathrm{~mm}$, et pour la zone d'immersion permanente une moyenne de $0.0012 \mathrm{~mm}$ et un écart-type de $0.141 \mathrm{~mm}$. Des études à venir analyseront ce point de manière plus détaillée. Les différences étant somme toute assez faibles et afin de ne pas surcharger l'analyse, on regroupe par la suite ces données. En s'intéressant aux distributions des erreurs de mesure pour chaque file comprenant un nombre de valeurs assez conséquent (files A, C, E et F), on constate que les distributions des erreurs de mesure sont proches avec un pic très net autour de la classe 0 .

\subsection{Comparaison des erreurs de mesure entre les deux postes}

Dans le même esprit que l'étude précédente, on compare ici les erreurs de mesure globale de chaque poste. On obtient des distributions identiques du type de celle présentée en figure 6 et les statistiques sont : pour le poste 2, une moyenne de $0.0015 \mathrm{~mm}$ et un écart-type de $0.1324 \mathrm{~mm}$; pour le poste 3 , une moyenne de $0.0018 \mathrm{~mm}$ et un écart-type de $0.1428 \mathrm{~mm}$. Ces données très proches semblent indiquer que les mesures effectuées l'ont été dans les mêmes conditions et que la répétabilité de la méthode semble bonne à entreprise donnée. On peut poser que l'incertitude sur la mesure se situe à $0.2 \mathrm{~mm}$.

\subsection{Modélisation probabiliste de la distribution de l'erreur de mesure}

On propose ici d'ajuster l'erreur de mesure par une loi standard. Prenons l'exemple du poste 2 : on présente sur la figure 6 la distribution de l'erreur de mesure du poste 2 avec deux ajustements (loi normale et de Student ou «t location-scale »). 


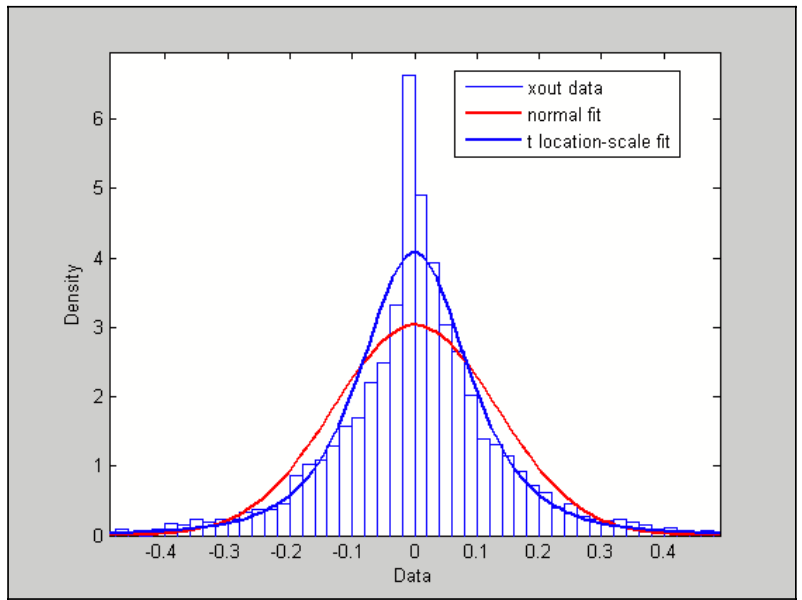

Figure 7 : lois d'ajustements de la distribution de l'erreur de mesure

On constate que la loi de Student donne un meilleur ajustement, bien qu'elle ne soit pas encore idéale : en effet si elle parait bien représenter la distribution de l'erreur sur les queues de distribution, elle ne correspond pas aux valeurs obtenues autour de zéro. De plus, tout comme la loi normale, la loi de Student n'est pas bornée ce qui est contraire à la réalité. Cependant aucune loi bornée, disponible dans la bibliothèque de Matlab (de type bêta par exemple), ne donne des résultats satisfaisants. Par aiellurs, pour les études ultérieures d'aide à la décision, seule la queues de distribution joue un rôle : c'est le cas de la construction des courbes R.O.C (Reciever Operating Characteristics).

Les paramètres des deux lois représentées sont les suivants: loi normale de moyenne $=9.7 \mathrm{E}-04 \mathrm{~mm}$ et d'écart type $=0.1314 \mathrm{~mm}$, et loi de Student de moyenne $=1.41 \mathrm{E}-17 \mathrm{~mm}$, d'écart type $=0.0903 \mathrm{~mm}$ et de degré de liberté $=3.2$. Enfin la loi normale présente un maximum de vraisemblance (en logarithme) égal à 1498 tandis que la loi de Student donne une valeur de 1606, montrant que la loi de Student ajuste mieux la distribution des erreurs de mesure.

\section{Conclusions}

Cet article exploite et compare des données des campagnes de mesures d'épaisseurs résiduelles de deux quais sur pieux similaires TMDC-2 et TMDC-3, situés dans l'estuaire de la Loire. Les pertes de matière, déduites à partir des épaisseurs nominales de chaque pieu, ont permis d'évaluer l'influence de la corrosion sur un peu plus de vingt ans de durée de vie des ouvrages. Cependant il a été mis en évidence que sans certitude sur les épaisseurs nominales des pieux, il est délicat de pouvoir utiliser ce type de mesure (problème des files A). A l'aide d'hypothèse sur ces épaisseurs, on a pu monter qu'il y avait de fortes similitudes dans le comportement face aux effets de la corrosion de deux ouvrages semblables (géométrie, âge, etc.). De plus nous avons pu mettre en évidence que l'erreur de mesure était très proche d'un site à l'autre et peut être caractérisée de manière intrinsèque, au moins à équipe d'inspection identique. Nous avons pu montrer qu'elle ne pouvait être ajustée par une densité de probabilité de type normale. 
Les perspectives se situent dans l'exploitation de campagnes expérimentales sur site afin de mettre à jour des modèles de cinétiques probabiliste présentés dans Clement1 se rapprochant plus dans un premier temps des modèles phénoménologiques de type Guedes-Soares ${ }^{2}$ que ceux paramétriques de type Melchers ${ }^{4}$.

\section{$\underline{\text { 6. Remerciements }}$}

Ce travail a été soutenu par une bourse d'études européenne et est financé par le Feder dans le cadre du projet Interreg IIIB intitulé «MEDACHS » (Marine Environment Damage to Atlantic Coast Historical and transport works or Structures). Les auteurs remercient le Port Autonome de Nantes Saint Nazaire et $\mathrm{N}$. Menard, pour la mise à disposition des données d'inspections des quais du TMDC ainsi que le CETMEF et plus particulièrement B. Benaïssa pour ces conseils et son intérêt.

\section{Références}

1 Clément A., Schoefs F., Memet J.B. (2006), «Steel report $n^{\circ} 3$ : Corrosion process modelling », MEDACHS, Marine Environment Damage to Atlantic Coast Historical and transport works or Structures. Programme européen Interreg IIIB.

2 Guedes Soares C., Garbatov Y. (1999), « Reliability of maintained corrosion protected plates subjected to non-linear corrosion and compressive loads », Marine Structures, Vol. 12, pp. 425-445.

3 Melchers R. E. (2003), « A new model for marine immersion corrosion in structural reliability assessment », Applications of Statistics and Probability in Civil Engineering.

4 Memet J.B. (2000) «La corrosion marine des structures métalliques portuaires : étude des mécanismes d'amorçage et de croissance des produits de corrosion ", Thèse de doctorat, Université de La Rochelle, $164 \mathrm{p}$.

5 Paik J. K., Lee J. M., Park Y., Hwang J. S., Kim C. W. (2003) « Time-variant ultimate longitudinal strength of corroded bulk carriers », Marine Structures, Vol. 16, pp. 567-600. 6 Rouhan A., Schoefs F. (2003), «Probabilistic modelling of inspection results for offshore structures », Structural Safety, Vol. 25, pp. 379-399.

7 Schoefs F., Capra B., Masson S. (2003), « Analyse de risque pour les ouvrages portuaires : cas de la perforation par corrosion d'un quai gabions ", VIII ${ }^{\text {èmes }}$ Journées Génie Civil - Génie Côtier, session posters, Compiègne 7-9 septembre 2004, Centre français du littoral, Tome 2, pp. 689-696.

8 Schoefs F., Clément A. (2004), "Multiple inspection modelling for decision and management of jacket offshore platforms : effects of false alarms », International Forum on Engineering Decision Making, December 5-9 2004, Stoos, Switzerland.

9 Straub D., Faber M. H. (2005), « Reliability updating for structures subject to localized corrosion defects », ICOSSAR 2005, Millpress, Rotterdam. 